\title{
Pushing the Limits of Local Excision for Rectal Cancer: Transanal Minimally Invasive Surgery (TAMIS) for Rectal Cancer Using the Port-in-Port Technique
}

\author{
Celia Robinson and Avo Artinyan \\ Michael E. DeBakey Department of Surgery, Baylor College of Medicine, Houston, TX
}

\begin{abstract}
Background. Radical rectal resection remains the standard of care for the operative treatment of rectal cancer. Local excision via transanal minimally invasive surgery (TAMIS) using disposable transanal access ports is an increasingly more common alternative for selected patients. Because of significant variation in perineal anatomy, currently available disposable transanal ports do not allow adequate access for every patient. This report demonstrates TAMIS for one such patient via a novel approach using a single-incision laparoscopic port inserted within a disposable transanal access port.

Methods. The patient is a 66-year-old man with a history of metastatic prostate cancer who was diagnosed with an anterior uT3N0 rectal cancer $9 \mathrm{~cm}$ from the anal verge occupying $40 \%$ of the rectal circumference. The patient refused radical resection and opted for multimodality therapy with local excision. He underwent preoperative chemoradiation with good tumor response. The TAMIS procedure was attempted and aborted due to poor visualization and inadequate access/exposure using the
\end{abstract}

commercially available Gelpoint Path transanal access system. The TAMIS procedure was reattempted and successfully completed via insertion of a laparoscopic singleincision port (GelPoint Mini) within the transanal access port itself (port-in-port technique) to overcome the deficiencies of the transanal access port. This report describes the port-in-port technique together with the stepwise approach to TAMIS.

Results. The TAMIS procedure was completed successfully without complications involving grossly negative margins. The patient's postoperative course was uncomplicated, and he was discharged on postoperative day 1 . The final pathology showed a complete pathologic response. At this writing, the patient continues to do well 14 months after surgery.

Conclusions. Currently available disposable transanal access ports may not allow adequate exposure for all patients undergoing TAMIS. This report describes a portin-port technique that may allow improved exposure for patients with a difficult perineal anatomy.

Electronic supplementary material The online version of this article (doi:10.1245/s10434-015-4978-1) contains supplementary material, which is available to authorized users.

(C) Society of Surgical Oncology 2015

First Received: 22 May 2015;

Published Online: 7 December 2015

A. Artinyan

e-mail: artinyan@bcm.edu 